\title{
The Impact of the Global Initiative for Asthma (GINA): Compass, Concepts, Controversies and Challenges
}

Helen K. Reddel, MBBS, PhD, FRACP

Woolcock Institute of Medical Research, University of Sydney, Sydney, Australia

\section{ABSTRACT}

The Global Initiative for Asthma (GINA) was established in 1993 to provide a global asthma strategy, adaptable to the needs of different countries. Over the past five years, GINA has initiated substantial changes in key concepts about asthma and its management, and in the structure and content of the GINA strategy report. These changes have sometimes generated controversy, but they have been welcomed in primary care, where most asthma is managed. The changes discussed in this review include a new definition of asthma that emphasises its heterogeneity; expansion of the scope of evidence considered by GINA, to give priority to both internal and external validity of research findings; and the integration throughout the report of practical advice about tailoring of treatment across the whole spectrum of asthma severity. The GINA report provides not only evidence-based recommendations, but also multiple practical resources for implementation in clinical practice. (BRN Rev. 2019;5(1):4-18)

Corresponding author: Helen K. Reddel, helen.reddel@sydney.edu.au

Key words: Asthma. Diagnosis. Phenotype. Practice Guideline. 
"No great advance has ever been made in science, politics, or religion, without controversy"

(Lyman Beecher, 1828) ${ }^{1}$

\section{BACKGROUND}

The Global Initiative for Asthma (GINA) was established in 1993 through a collaboration between the National Heart, Lung, and Blood Institute (NHLBI) and the World Health Organization. It was tasked with providing an asthma strategy that could be adapted to the health context and healthcare systems in different countries. The first GINA Global Strategy for Asthma Management and Prevention was published in 1995. Since 2002, GINA has published annual updates based on a twice-yearly incremental review of asthma literature, with major revisions in 2006 and 2014; the current GINA report was published in February $2018^{2}$. Dissemination of the GINA strategy report is supported by the efforts of members of the GINA Assembly, currently representing 45 countries, and by activities associated with World Asthma Day, which was established by GINA in 1998 and is celebrated on the first Tuesday in May.

Although the GINA report is often colloquially referred to as "international asthma guidelines", GINA itself describes it as a global strategy, that can be adapted by local experts into guidelines tailored for the local healthcare system, regulatory framework, and medication availability ${ }^{3}$. A 2013 survey by the International Primary Care Respiratory Group (IPCRG) of its member countries reported that the GINA report was used by clinicians in $14 / 21$ countries. In addition, of 14 national asthma guidelines, 10 had been based on or informed by the GINA report ${ }^{4}$. A 2013 survey of clinicians in 92 countries by the Global Asthma Network found that the GINA report was used exclusively in $17 \%$ of countries, and a further $25 \%$ of countries used several types of guidelines including GINA; $60 \%$ of countries did not have national asthma guidelines ${ }^{5}$.

The broad international compass of GINA can also be seen from the dissemination of its resources. For example, in the first eight months of 2018, the GINA website received over 260,000 unique visitors, with the top 10 countries of origin being: USA, India, Brazil, Malaysia, Mexico, Thailand, United Kingdom, Indonesia, Colombia and the Philippines. In the same period, over 250,000 free electronic copies of 2018 GINA resources were downloaded from the website, including 160,000 full reports, 75,000 Pocket Guides (English, French, German, Hebrew, Mandarin; with an additional 70,000 copies purchased in print), and 17,000 copies of the 2018 GINA slide set. A further 150,000 copies of GINA resources were downloaded from countries behind privacy firewalls.

Since its inception, GINA has initiated substantial changes in key concepts about asthma and its treatment that have had a substantial impact on asthma management globally, although these changes have not been without controversy. This article describes several key areas of change that have been implemented by GINA in recent years, with a discussion about the rationale for each and the debate that followed, identifying important evidence gaps. The tables summarise key messages (Table 1), key areas of change in the past few years (Table 2), and current controversies (Table 3). 
TABLE 1. Key messages

Make the diagnosis of asthma early, if possible before controller treatment is started, based on a typical pattern of variable respiratory symptoms and one or more tests for variable expiratory airflow limitation

At every visit, ask about symptom control in the last four weeks, flare-ups (exacerbations, attacks) in the last year, and how often reliever and controller medications are being taken

Personalise treatment decisions by also taking into account each patient's specific risk factors for exacerbations, their comorbidities and their goals and preferences

Most patients with asthma should take at least low-dose inhaled corticosteroids (ICS) as this greatly reduces the risk of severe exacerbations and asthma-related death

Never treat patients with asthma or a history of asthma with long-acting $\beta_{2}$-agonists (LABA) without ICS

Check and correct poor adherence and incorrect inhaler technique before considering a step-up of controller medication, as these are common causes of poorly-controlled asthma

Train patients in asthma self-management, with monitoring of symptoms and/or lung function, a written asthma action plan, and regular medical review, so they know how to respond to worsening asthma

Refer the patient for specialist review early if there is difficulty confirming the diagnosis of asthma, if occupational asthma is suspected, or if the patient has persistent symptoms and/or exacerbations despite correct technique and good adherence with moderate or high dose ICS/LABA

Good communication is essential. Establish a partnership with the patient, taking into account their health literacy, personal goals and fears, and cultural issues

TABLE 2. Changes in concepts in the past five years

Asthma is a heterogeneous condition, usually characterised by airway inflammation. There are likely to be several different underlying mechanisms

Patients with good asthma symptom control or infrequent symptoms may still be at risk of severe exacerbations or asthma-related death. Modifiable risk factors should also be assessed

Asthma treatment is not one-size-fits-all, but is based on an individualised assessment of each patient's symptoms, modifiable risk factors including comorbidities and environmental exposures, their lung function, and their personal preferences

Very few if any patients should now be prescribed short-acting $\beta_{2}$-agonist reliever alone, without inhaled corticosteroids (ICS); at present, this should only be considered for patients with symptoms less than twice a month, no night waking due to asthma, and no risk factors for exacerbations

Asthma severity is a retrospective label, assessed from treatment needed to control asthma

An early rapid increase in ICS is recommended for asthma action plans, with the greatest reduction in exacerbations provided by the low-dose ICS/formoterol maintenance and reliever regimen

An increasing number of biologic therapies are available for treatment by subcutaneous injection for patients with asthma that is relatively refractory to conventional inhaled treatment

In acute asthma, recommendations for oxygen therapy have been revised, with establishment of target upper level for oxygen saturation (95\% for adults, $98 \%$ for children)

Asthma and chronic obstructive pulmonary disease (COPD) are not separate diseases that may co-exist, but they are overlapping heterogeneous conditions; further research is needed to identify underlying mechanisms

\section{REDEFINING ASTHMA}

Concepts of asthma have changed substantially over the past 50 years. Figure 1 shows an archival illustration created by the late
Professor Ann Woolcock, one of the founding members of GINA, illustrating the swing of thinking about mechanisms from 1968 (a disease of bronchoconstriction) to mid-1990s (a disease of inflammation), then by 2000 (the 


\section{TABLE 3. Current controversies}

Can asthma be prevented, in children and in adults? Although there are several known associations with development of asthma, there have been no successful interventions

Should any asthma patients be prescribed short-acting $\beta_{2}$-agonist (SABA) alone, without any inhaled corticosteroids (ICS)? Although SABAs are effective in treating symptoms, they do not treat asthma itself, and with over-use, they increase rather than decrease the risk of exacerbations

Are there any biomarkers that can identify patients with asthma who can safely be treated without any ICS, or in whom ICS can safely be stopped? Although an elevated exhaled nitric oxide level identifies patients who have a short-term benefit from ICS treatment, there are no long-term studies evaluating safety of treating without ICS; and several important triggers for eosinophilic asthma exacerbations are variable and unpredictable in their occurrence (e.g., respiratory viruses, air pollution, and aeroallergens).

Are there any biomarkers that can be used to adjust long-term asthma treatment in adults? Although adjustment of treatment by exhaled nitric oxide level has been shown to reduce exacerbations in children compared with current guidelines-based adjustment, the same has not been found in adults (see Petsky et al. Analyses 1.10 and $1.11^{61}$ ). Existing markers of type 2 inflammation (e.g., blood eosinophils, exhaled nitric oxide level) are not stable enough to satisfy regulators' current analytical validation criteria for disease biomarkers.

How should seasonal asthma be treated in patients who have no out-of-season symptoms? Seasonal treatment with ICS is often implemented for such patients in clinical practice, but there is no specific evidence.

Can patients who are relatively refractory to ICS treatment be identified early in the course of the disease, so they can be fast-tracked to specialist care, rather than waiting to identify failure of response to moderate or high dose ICS-containing treatment?

Do small-particle ICS achieve better clinical outcomes than larger-particle ICS? Although this has been widely supposed on theoretical grounds, there are no good quality randomised controlled trials.

How can the cost-effectiveness of biologic therapy be improved? Would use of a biologic therapy early in the lifetime of asthma change the course of the disease?

How can accelerated decline in lung function or development of persistent airflow limitation be prevented in patients with asthma?

A Ann Woolcock - the asthma pendulum (1993)

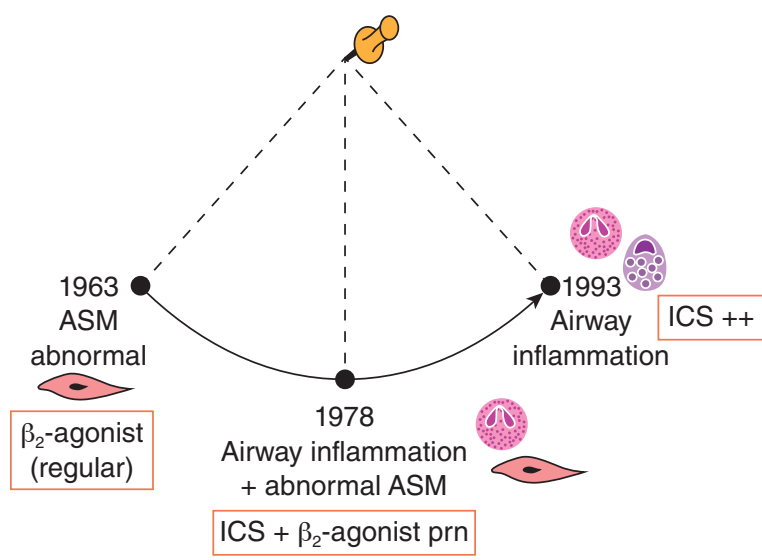

B Ann Woolcock - the asthma pendulum (2000)

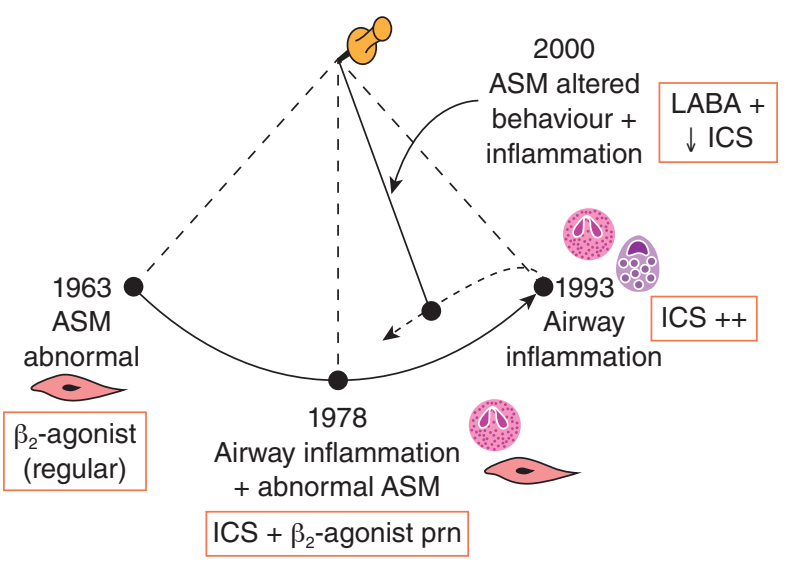

FIGURE 1. The figure shows archival figures from the late Professor Ann Woolcock, one of the founding members of GINA (reproduced with permission). A: In 1993, she showed the swing of thinking from 1968, when asthma was considered a disease of bronchoconstriction and treated with regular SABA, to the late 1970s when eosinophilic airway inflammation was well-recognised and treatment shifted to regular ICS and as-needed SABA, then to the mid-1990s, when the focus was primarily on airway inflammation (which, in this image, was already illustrated as heterogeneous), and treatment with high dose ICS was recommended. B: Professor Woolcock updated the slide in 2000, the year before her untimely death, to show the pendulum swinging back to acknowledge the role of both inflammation and abnormal airway smooth muscle, and with treatment including LABAs and lower doses of ICS.

ASM: airway smooth muscle; GINA: Global Initiative for Asthma; ICS: inhaled corticosteroids; LABA: long-acting $\beta_{2}$-agonist; SABA: short-acting $\beta_{2}$-agonist. 
year before Professor Woolcock's untimely death) swinging back to acknowledge the role of both inflammation and abnormal airway smooth muscle.

By 2014, the widely-used definition of asthma comprised a lengthy description of a disease characterised by chronic inflammatory cells, airway hyperresponsiveness (AHR), variable airflow limitation, and variable symptoms. There was increasing evidence of heterogeneity, with recognition of clinical and, later, inflammatory phenotypes. This indicated the potential to identify specific underlying mechanisms and opportunities for targeted treatment. In addition, there was increasing evidence that asthma was both under- and over-diagnosed $^{6}$, indicating the need for greater emphasis on diagnostic criteria.

A key priority for GINA in 2014 was therefore to provide a definition of asthma that reflected the complexity of asthma as a heterogeneous disease, that would accommodate emerging evidence of different inflammatory and pathophysiological mechanisms contributing to the cardinal features of variable respiratory symptoms and variable expiratory airflow limitation, and would avoid previous assumptions about the mechanistic relationship between inflammation, AHR, symptoms and exacerbations; the limitations of these assumptions were elegantly described by Anderson 7 . Importantly, the definition should also be feasible for use in diagnosing asthma in clinical practice. The new definition was: "Asthma is a heterogeneous disease, usually characterised by chronic airway inflammation. It is defined by the history of respiratory symptoms such as wheeze, shortness of breath, chest tightness and cough that vary over time and in intensity, together with variable expiratory airflow limitation." The accompanying text highlighted that some patients with asthma later developed persistent airflow limitation.

\section{Concept: should the label 'asthma' be abandoned?}

Controversies surrounding this definition included whether the term "asthma" should be abandoned, given its known heterogeneity; whether airway inflammation should be an obligatory component of the asthma definition; and whether variable airflow limitation was an essential component. Regarding the term "asthma", GINA now explicitly emphasises the heterogeneity of asthma and uses the term "asthma" as an "umbrella term" like "anaemia", "arthritis" and "breast cancer". Such terms are important in communication with patients and for advocacy, and they facilitate recognition in primary care of heterogeneous diseases that have readily recognisable clinical features in common; their use carries no implication that treatment will be "onesize-fits-all". However, in asthma, by contrast with anaemia, arthritis and cancer, evidence about underlying mechanisms is far less well-established ${ }^{8}$. Many studies of underlying inflammatory pathways are only in patients with severe asthma, and the severity categories themselves are still retrospective labels, dependent on the clinician having excluded other contributory causes and demonstrated (relative) refractoriness to conventional inhaled treatment. Further research in broader populations is clearly needed, to develop objective markers of underlying mechanisms and severity that are independent of treatment. However, in the meantime, community studies have 
shown that the label "asthma" (regardless of how assigned) carries an important safety signal, identifying patients with an increased risk of hospitalisation or death if not treated with inhaled corticosteroids (ICS) ${ }^{9}$. These risks have been documented in younger age groups ${ }^{10}$, in patients treated with separate ICS and long-acting $\beta_{2}$-agonist (LABA) inhalers ${ }^{11}$ and in patients with diagnoses of both asthma and chronic obstructive pulmonary disease (COPD), prescribed LABA-only treatment ${ }^{12}$.

The words "usually characterized by chronic airway inflammation" in the asthma definition raised some concerns that this downplayed the importance of anti-inflammatory treatment. However, the rationale for the word "usually" was that there was insufficient evidence to rule out asthma if a patient lacked currently available inflammatory markers; and the new definition was accompanied by a greater - rather than lesser - emphasis on the importance of ICS.

\section{BROADENING THE SCOPE OF EVIDENCE}

For diagnosis and management of diseases such as asthma, health professionals rely on evidence-based recommendations, including those developed as formal clinical practice guidelines. Extensive work has been carried out on guidelines methodology, including formulation of clinical questions in Population, Intervention, Comparison, Outcomes (PICO) style, with systematic reviews for each question, followed by evaluation of the quality and strength of evidence using approaches such as Grading of Recommendations, Assessment, Development, and Evaluation (GRADE) ${ }^{13}$. The number of questions may be very large: for example, 350 questions were identified in scoping for new Australian asthma guidelines ${ }^{14}$. Further, different bodies may make different recommendations from the same evidence, as was seen recently in the United Kingdom in asthma guidelines published by the British Thoracic Society/Scottish Intercollegiate Guideline Network (BTS/SIGN) and the National Institute for Health and Care Excellence $(\mathrm{NICE})^{15}$. The length and cost of the formal guidelines development process has led to a longer interval between updates, and is understood to have led many guidelines developers to reduce the scope of their guidelines $^{16}$. The reduction of guidelines to answers to a small set of individual questions also leaves clinicians without an overall strategy for managing complex chronic conditions such as asthma. A recent investigation by the American Thoracic Society found that a consensus-based process greatly shortened the time to reach concordant recommendations, by reducing the number of questions for which formal systematic review was needed ${ }^{16}$, but this requires further evaluation ${ }^{17}$. In the meantime, health professionals and patients increasingly source health information online, with little awareness that the information may be out of date, or specific to a particular country, healthcare system or regulatory framework.

In order to be considered for clinical recommendations, a therapy obviously needs to be efficacious, i.e. to work under ideal circumstances. This is established with highly controlled clinical trials, often carried out for regulatory purposes, usually double blind and placebo-controlled to minimise the potential for bias and to maximise internal validity. In GINA, before a recommendation for a new 
medication is made, evidence of efficacy is required from at least two good quality clinical trials, plus an indication for asthma by a major regulator. The latter requirement provides an important safety check, since the regulatory agencies may see a much larger dossier of evidence for new medications than is published in peer-reviewed literature; an indication by a major regulator also ensures that a large number of clinicians will be able to prescribe the medication.

The formal guidelines process described above places a very high value on internal validity ${ }^{13}$, but a low value on the generalisability of the evidence to community patients. Populations included in efficacy studies are highly selected, with few comorbidities; participants are usually required to attend multiple visits, and to be highly adherent with study medications, questionnaires and diaries. Clinical practice involves much broader populations, with many patients having comorbidities, poor socioeconomic circumstances, low health literacy and ongoing environmental exposures, or who do not satisfy the criteria for "classic" asthma. This may include having a history of smoking, or persistent airflow limitation from long-standing asthma. In 2007, it was estimated that a median of only $5 \%$ of asthma patients from a random population sample would have satisfied the entry criteria for 17 major studies ${ }^{18}$. This brings into question the generalisability and safety of recommendations made on the basis only of efficacy studies.

In 2014, therefore, GINA made a deliberate decision to evaluate a broader range of evidence than only efficacy studies. Where relevant, the Scientific Committee now also considers pragmatic studies and even observational studies, to fill evidence gaps for specific populations, and to understand heterogeneity in treatment response, although still with evaluation of potential bias. A key design limitation that we have noted in most observational studies is the lack of adjustment for the prescribing physician/practice, which effectively (but implausibly) assumes that the decision of the physician to prescribe medication A rather than medication $B$ at one point in time is independent of every other clinical decision they make about that patient's asthma management during the follow-up period. For efficacy studies, greater attention is being paid to the study populations, to identify factors that may be relevant to the interpretation of findings ${ }^{19}$.

In many cases, the results of efficacy and effectiveness studies are concordant, with the main differences being in the magnitude of benefit, since interventions in efficacy studies are generally studied under ideal conditions. However, two examples are given below in which the consideration of a broader range of evidence has allowed GINA to provide interim recommendations in the absence of a relevant efficacy study, or to modify recommendations based only on efficacy studies.

\section{Concept: asthma-chronic obstructive pulmonary disease overlap}

One of the most characteristic features of efficacy studies in asthma and COPD is their restriction to "pure" asthma and "pure" COPD, excluding patients with both diagnoses, patients with asthma who are current smokers, have a $>10$ pack-year smoking history and/or have developed persistent airflow limitation, and patients with COPD who have 
a past history of asthma ${ }^{18,20}$. This reflects the historical distinction made between asthma and COPD in clinical guidelines in 199521, and specific regulatory requirements. However, such exclusion criteria have persisted despite increasing recognition that many patients with asthma or COPD (15-60\% of those with either diagnosis ${ }^{22,23}$ ) have features of both conditions. Importantly, these patients differ from those with either diagnosis alone, in having worse quality of life, higher mortality, and greater healthcare utilisation ${ }^{22-24}$, and several studies have reported that they have more comorbidities than patients with COPD alone ${ }^{25}$. Therapeutic recommendations based only on efficacy studies thus lack relevance to a large proportion of patients with airways disease in clinical practice.

This issue has far more serious implications than just a lack of evidence, because current asthma and COPD guidelines provide directly contradictory safety recommendations about treatment, based on high quality evidence from efficacy studies ${ }^{23}$. For COPD, treatment starts with long-acting bronchodilators (LABA and/or long-acting muscarinic antagonists [LAMA]) alone; because of concern about the risk of pneumonia with ICS, use of ICS is limited to patients with persisting exacerbations and low lung function. By contrast, in asthma guidelines, there are strong safety warnings about the risks of treating asthma with LABA alone, because of the risk of severe exacerbations and death. What is the primary care clinician to do, when treating a patient who does not have "pure" asthma or COPD?

In response to these issues, and recognising that overlapping features of asthma and COPD would likely result from a range of different underlying mechanisms, GINA collaborated with the Global Initiative for Obstructive Lung Disease (GOLD) to develop interim practical advice for clinicians, particularly generalists and those in primary care. This included how to recognise typical asthma and typical COPD, and pragmatic safety-based recommendations including that patients with features or asthma or a diagnosis of asthma should at least receive low-dose ICS. Evidence was severely limited ${ }^{26}$, but it included that in a very large case-control study of community patients with asthma (not excluding smokers or those with a diagnosis of COPD), low dose ICS reduced the risk of asthma-related death by $85 \%{ }^{10}$, and reduced the risk of asthma-related hospitalisation by $31 \%$ and of rehospitalisation by $39 \% 26,27$. This interim advice is now further supported by a well-conducted case control study in patients with newly-diagnosed COPD, with death or hospitalisation as the outcome ${ }^{12}$, and by the findings of the UK National Review of Asthma Deaths ${ }^{9}$.

Interestingly, introduction of this interim safety-based advice was followed by substantial controversy, appearing largely due to an (incorrect) assumption that the term asthma-COPD overlap syndrome ${ }^{28}$, later shortened to ACOS, implied a single disease. Much of this controversy likely resulted from two different meanings of "syndrome": its conventional medical usage refers to a cluster of symptoms and signs that may have different underlying causes (e.g., Cushing syndrome), but some commentators restrict the term "syndrome" to conditions that have a single underlying mechanism (e.g., Klinefelter syndrome ${ }^{29}$ ). To reduce confusion, the word "syndrome" was removed in 2017, with re-emphasis of the 
need for research to fill the obvious evidence gaps about causes and treatment.

\section{Concept: asthma action plans, and the importance of background adherence}

One of the key differences between patients in efficacy studies and those in clinical practice is in their adherence with prescribed medication. Adherence in regulatory asthma studies is typically $85-95 \%$, whereas in clinical practice, it is typically $25-35 \%{ }^{30,31}$. Fewer than half of asthma patients fill more than one maintenance prescription in a year, and only a small proportion has enough dispensing to be consistent with semi-regular use $\mathrm{e}^{31,32}$. By contrast, patients use ICS-containing medication irregularly, relying on their short-acting $\beta_{2}$-agonist (SABA) reliever medication instead, including when asthma worsens ${ }^{33}$.

Such evidence has important implications for recommendations about worsening asthma. All guidelines recommend that patients should have a written asthma action plan, but following the publication of two large well-conducted placebo-controlled studies in 2004 34,35 , guidelines changed to recommend against doubling the dose of ICS in the "yellow zone", instead advising only increased SABA use until the patient was sick enough to need oral glucocorticoids. In 2014, GINA reconsidered a broad range of evidence relevant to these recommendations in adults ${ }^{8}$. This included that most exacerbations are associated with increased inflammation ${ }^{36}$; that meta-analysis of action plans involving doubling of ICS as well as addition of oral glucocorticoids showed improved health outcomes ${ }^{37}$; evidence for the benefit from quadrupling the dose of ICS or budesonide/formoterol in adults ${ }^{38}$; and the evidence from both efficacy and effectiveness studies for reduction of exacerbations with an early small increase in ICS/formoterol dose with maintenance and reliever treatment $(\mathrm{MART})^{39}$. The generalisability of the two placebo-controlled efficacy trials was limited by their highly selected populations ${ }^{34,35}$, by obligatory high adherence, and by delayed initiation of the increased dose. The conclusion that can be drawn is that, for patients with high adherence $\left(>95 \%{ }^{34}\right)$, doubling of ICS after asthma worsens is not efficacious. However, the proportion of community patients with such high adherence is vanishingly small ${ }^{30,31}$; this issue is particularly important given the very steep dose response curve for $\mathrm{ICS}^{40}$. For clinical practice, therefore, GINA 2014 provided specific recommendations about action plans for different controller regimens, including the small increases in dose with the MART regimen, and suggesting that, for patients on maintenance regimens, clinicians should consider at least doubling ICS in the "yellow zone" of their action plan. The discussion will continue following recent publication of additional efficacy and effectiveness studies ${ }^{41,42}$.

\section{TARGETING TREATMENT TO REDUCE RISK}

A major change in GINA 2014 was its focus on targeted treatment. This was not restricted to biologic therapy for severe asthma, but advice was provided about tailoring treatment for individual patients across the whole spectrum of asthma severity, with a particular focus on reducing the risk of exacerbations. Personalised treatment was integrated throughout 

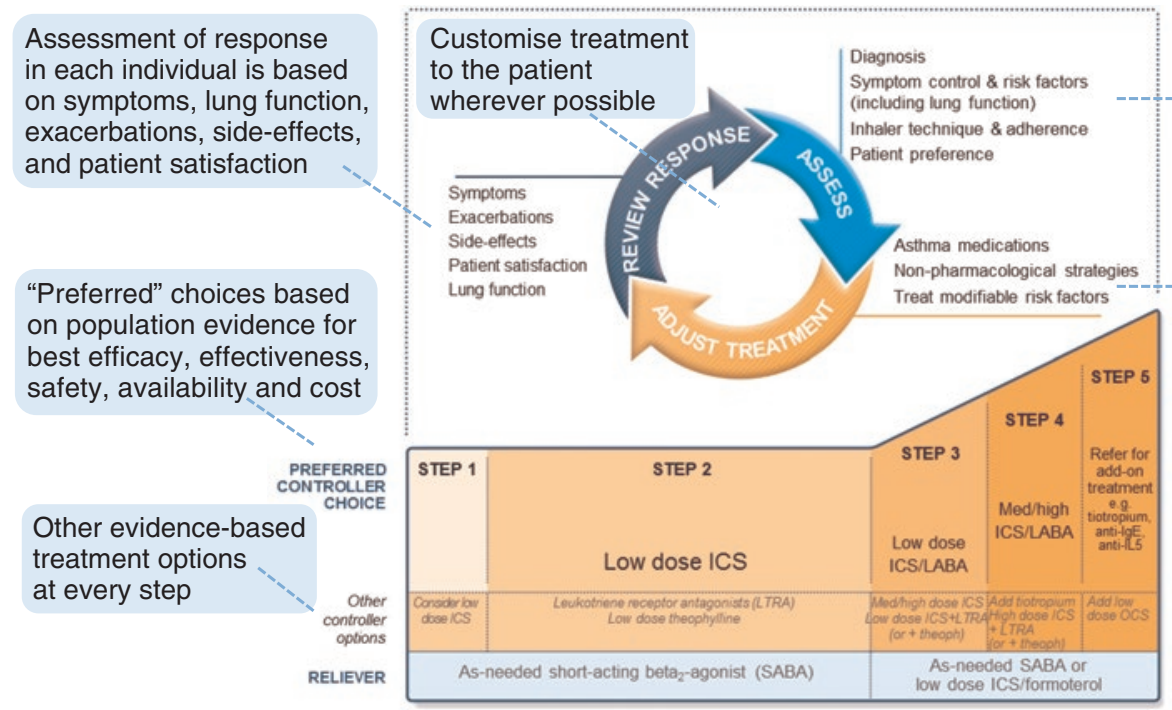

Individual treatment choices are based on symptoms, risk factors (e.g. $F E V_{1}$, smoking, FeNO, exposures etc) and patient's perspective. Before stepping up, first check for common problems

Not just drugs: also treat modifiable risk factors and comorbidities; use non-pharmacological strategies where relevant

Figure 2. The main part of the GINA treatment figure (Box 3-5, GINA 2018), annotated to highlight how personalised treatment is integrated throughout asthma management, across the range of severity (CCopyright GINA, modified with permission of GINA). FeNO: fractional concentration of exhaled nitric oxide; FEV $_{1}$ : forced expiratory volume in one second; ICS: inhaled corticosteroids; IgE: immunoglobulin E; IL: interleukin; LABA: long-acting $\beta_{2}$-agonist; OCS: oral glucocorticoids; theoph: theophylline.

the report, including in the main treatment figure (Fig. 2), representing a major change from the "one-size-fits-all" concepts in other guidelines.

A clear distinction is drawn by GINA between treatment decisions made at a population level (e.g., national guidelines, health maintenance organisations), in which the treatment which would be best for most patients is based on group mean data for symptoms, exacerbations and lung function, plus availability and cost; and patient level treatment decisions, which should also take into account: (a) any patient characteristic or phenotype that predicts differences in their risk of adverse outcomes or their response to treatment, or any modifiable risk factors or comorbidities that might be contributing to symptoms or exacerbations; (b) patient preference; and (c) practical issues such as inhaler technique, adherence, and affordability. These criteria reflect the importance GINA places on individualising patient management not only by using biomarkers, but also by incorporating "humanomics", i.e. considering the behavioural, social and cultural factors that are relevant to individual patients ${ }^{43}$.

\section{Concept: what factors can be used in tailoring treatment of asthma?}

To facilitate personalised treatment decisions, GINA compiled a list of modifiable risk factors that would prompt specific treatment, either pharmacological or non-pharmacological. Poor symptom control is such a strong 
predictor of exacerbations that risk factors were only listed if they remained significant after adjustment for symptoms, i.e. the presence of any of these risk factors increased the patient's risk of exacerbations even if they had few or no symptoms. This criterion is particularly important for biomarkers that involve cost or inconvenience (e.g., blood sampling), since these will only be worthwhile if they provide information additional to that which can be obtained by asking simple questions about symptom control. For patients with $\geq 1$ risk factor for exacerbations, basic strategies include: optimising ICS therapy to minimise exacerbation risk, including MART where available (not in the US); ensuring that the patient has a written action plan appropriate for their health literacy; reviewing the patient more frequently than low-risk patients; and checking inhaler technique and adherence frequently; as well as more specifically tailoring therapy by identifying any personal or environmental risk factors that can be treated or modified.

There is substantial interest in combining risk factors into a risk score for clinical practice. In one study, the modifiable risk factors listed in GINA 2014 were used in development and external validation of risk prediction mod$\mathrm{els}^{44}$. The history-related items from the GINA list that remained significant in the models were Asthma Control Questionnaire (ACQ) score, current smoking, chronic sinusitis, previous hospital admission for asthma and $\geq 1$ severe exacerbation in the previous year. This model gave an area under the curve (AUC) for exacerbations of 0.77 (95\% confidence interval [CI] 0.75 to 0.80 ). Addition of spirometry, or spirometry and fractional concentration of exhaled nitric oxide (FeNO) provided only slightly better discrimination, with AUC of 0.79 and 0.80 respectively ${ }^{44}$. Further evidence is needed in broader populations ${ }^{45}$.

Unfortunately, in airways disease, there has been a noticeable lack of routinely-collected data and hard outcomes, by comparison with the data available for development of the risk scores for cardiovascular disease that have guided treatment. Sputum induction provides the gold standard for evaluation of airway inflammation, and in secondary care patients with moderate-to-severe asthma, sputum-guided treatment is superior to guidelines-based treatment ${ }^{46,47}$. However, implementation of sputum analysis in clinical practice has generally proved feasible only in specialist centres that also have a high throughput of research patients. Initially, it was assumed that assessment of FeNO may provide a suitable substitute, but the association of FeNO with sputum eosinophils proved to be only moderate $^{48}$. In adults to date, only three studies have compared FeNO-guided treatment with guidelines-based treatment, and none showed a significant advantage for exacerbations ${ }^{47}$. Studies in which a range of other comparator algorithms were used are of interest, but they do not help to answer the question of whether FeNO-guided treatment is associated with better outcomes than current treatment recommendations.

One study of FeNO that may be of particular utility was conducted in patients with difficult-to-treat asthma with high baseline $\mathrm{FeNO}^{49}$. Those whose FeNO decreased within 5 days of directly-observed ICS therapy were found, based on dispensing data, to have previously been poorly adherent ${ }^{49}$. Given the high cost of biologic therapy for severe asthma, 
a FeNO suppression test could prove cost-effective in distinguishing between asthma that is difficult to treat because of problems with adherence and/or inhaler technique, and asthma that is relatively refractory to ICS. The rapidity of the change in FeNO in this study suggests that the well-recognised phenomenon of "white-coat adherence", in which patients take more medication than usual in the week before and after a clinic visit, could affect the utility of FeNO in assessing asthma in clinical practice.

\section{Concept: which asthma patients should be prescribed inhaled corticorteroids?}

A review of evidence in preparation for GINA 2014 identified that evidence was lacking for the then-standard criteria for starting ICS, e.g., symptoms $>2$ days a week. These criteria appeared to have been carried forward without challenge from the first asthma guidelines. A pre-specified post hoc analysis of the long-term Inhaled Steroid Treatment as Regular Therapy in Early Asthma (START) study ${ }^{50}$ was arranged, demonstrating that the risk of serious exacerbations was halved by low-dose ICS even in patients with so-called "intermittent asthma" ${ }^{\prime 51}$. Because of this, the known risk of severe exacerbations and asthma-related death even in patients with mild or infrequent symptoms ${ }^{52}$, and the lack of safety data for SABA-only treatment, GINA restricted the latter to patients with symptoms less than twice/month, no night waking, and none of a long list of risk factors for exacerbations. Future discussions will examine the potential role of as-needed budesonide/formoterol in reducing exacerbations in mild asthma ${ }^{53,54}$.
There is interest in whether FeNO could be used to predict if patients with respiratory symptoms would respond to ICS. There have been two short-term studies ${ }^{55,56}$, but there is no evidence that it is safe (with regard to risk of exacerbations) to withhold ICS in patients with a low baseline FeNO. The need for longterm studies is particularly important, given the variability of FeNO in response to external agents such as allergens, viral infections, and pollution that themselves may be unpredictable in timing. In addition, symptoms of eosinophilic bronchitis - which is also characterised by elevated FeNO - respond to short-term treatment with ICS, but eosinophilic bronchitis rarely progresses to asthma and carries no risk of serious exacerbations $\mathrm{s}^{57}$.

\section{PRACTICAL RESOURCES}

A key feature of GINA reports over the past 5 years, based on feedback from primary care clinicians and allied health professionals, has been the clinically-focussed content, not only presenting evidence-based content about what should be done, but also with multiple practical flow charts and summary tables about how to implement the recommendations, in order to improve utility for busy clinicians. An assumption has been made that clinicians will dip in and out of the report to answer specific clinical questions, rather than reading it from cover to cover, so key messages such as inhaler technique and adherence are repeated wherever relevant, rather than assuming that the reader will have seen this elsewhere. For example, a review of the GINA 2015 report found that $36.7 \%$ of figures and tables had content about inhaler devices, compared with an average of $<3 \%$ for other asthma and COPD 
guidelines $^{58}$. This new style of report has been particularly welcomed in primary care where most patients with asthma are managed.

Asthma management is becoming more complex, with biologic therapies providing increased treatment options for patients whose asthma is relatively refractory to conventional inhaled therapy. However, the extremely high cost of these treatments, and a lack of specialist resources in many countries, provide incentives to effectively identify and manage common issues such as inhaler technique and adherence in clinical practice, that otherwise contribute substantially to poor symptom control, exacerbations and asthma-related mortality. Given the global scope of GINA, treatment options that are suitable for developing countries are also a priority.

\section{ChALlenges}

Developing evidence-based recommendations for clinical practice is acknowledged as being a complex, costly, and time-consuming process, and for most national guidelines there is a lag of several years between updates. GINA is faced with major challenges in maintaining an annually updated strategy report. The evidence available from randomised controlled trials, observational studies and systematic reviews is rapidly expanding, increasing the workload for members. GINA does not have resources to fund its own systematic reviews, so there are some persisting gaps in evidence. In order to provide a global strategy, GINA needs to evaluate therapeutic options that are relevant to low- and high-income countries; even within higher-income regions, there is substantial variation in population characteristics, healthcare systems, medication availability and funding. It is therefore essential also to have national or regional guidelines, in order to provide locally-specific recommendations. The work of GINA is supported only by the sales and licensing of its products, but GINA products will continue to be made available for free download by individual health professionals.

\section{FUTURE DIRECTIONS}

There is keen interest by GINA in expanding understanding of the mechanisms underlying different asthma phenotypes and identifying specific treatment strategies for each. For progress to be made, mechanistic and observational studies need to include patients from across the whole spectrum of airways disease rather than being limited to the conventional disease labels of "asthma" and "COPD". The study of underlying mechanisms in diseases of the airways and lungs, although high in quality, is decades behind that in conditions such as cancer, cardiovascular disease and diabetes, where objective measures have been consistently measured and recorded. Evidence emerging from studies such as the Unbiased Biomarkers for the Prediction of Respiratory Disease Outcomes (UBIOPRED) study suggests that, for respiratory disease, multidimensional tools such as breathomics $^{59}$ or proteomics may provide the most radical advances in knowledge; ideally, measures such as these should be included in every clinical trial, but cost and standardisation remain important barriers.

In the meantime, yearly updates provide the opportunity for rapid incorporation of new 
medications and new treatment approaches into the strategy report, as evidence from new clinical trials and systematic reviews emerges and is evaluated. This is particularly important for severe asthma, the prevalence of which is estimated at only $3.7 \%$ of the asthma population $^{60}$, but for which there is a rapidly-expanding suite of biologic therapies that can markedly reduce severe exacerbations and improve quality of life. Given the high cost of these therapies, it is important to target their use to patients whose asthma is refractory or relatively refractory to conventional inhaled therapies. To this end, GINA will shortly launch a new pocket guide for assessment and management of patients with difficult-to-treat and severe asthma. This resource, which has been developed through extensive collaboration with experts in human-centred design, provides a decision tree and practical advice, including about choice between and use of available biologics. The lack of direct head-tohead studies remains a problem, and readers need to take into account local eligibility requirements, which vary from payer to payer.

Collaborations are needed with key stakeholder bodies and respiratory organisations, particularly in primary care, to identify the most appropriate research questions and study designs to fill current knowledge gaps, and to provide opportunities to translate the findings into clinical recommendations and implement them into clinical practice globally.

\section{DISCLOSURES}

The author or her institute have received honoraria for providing independent medical education at symposia funded by AstraZeneca,
GlaxoSmithKline, Novartis, Teva, Mundipharma, Boehringer Ingelheim; for data safety monitoring boards and/or advisory boards for AstraZeneca, GlaxoSmithKline, Merck, Novartis, Boehringer Ingelheim, and for consulting for AstraZeneca and GlaxoSmithKline. Her institute has received independent research grants from AstraZeneca and GlaxoSmithKline.

The author is Chair of the Global Initiative for Asthma (GINA) Scientific Committee. This is an honorary position. The work of GINA is supported solely by the sale and licensing of its resources.

\section{REFERENCES}

1. Beecher L. Sermons Delivered on Various Occasions (1828). 1852: John P. Jewett \& Company (republished by Google Books).

2. Global Initiative for Asthma. Global strategy for asthma management and prevention. Updated 2018. 2018. Available from: www.ginaasthma.org.

3. The ADAPTE collaboration (2009). The ADAPTE Process: Resource Toolkit for Guideline Adaptation. Version 2.0. Available from http:/ / www.g-i-n.net

4. International Primary Care Respiratory Group. Mapping of national guidelines used by primary care. 2013. Available from: https://www.theipcrg. org / display / ResMapping / Mapping+of+national+guidelines+ used+by+primary+care. Accessed 7 Sept 2018.

5. Global Asthma Network, The Global Asthma Report 2014. 2014: Auckland, New Zealand.

6. Aaron SD, Boulet LP, Reddel HK, Gershon A. Under-diagnosis and over-diagnosis of asthma. Am J Respir Crit Care Med. 2018;198:1012-20

7. Anderson GP. Endotyping asthma: new insights into key pathogenic mechanisms in a complex, heterogeneous disease. Lancet. 2008;372:1107-19.

8. Reddel HK, Bateman ED, Becker A et al., A summary of the new GINA strategy: a roadmap to asthma control. Eur Respir J. 2015;46:622-39.

9. Levy ML, Andrews R, Buckingham R, Evans H, Francis C, Houston R, et al. Confidential Enquiry report. London: Royal College of Physicians; 2014. Why asthma still kills: The National Review of Asthma Deaths (NRAD) Available online from: www.rcplondon.ac.uk/sites/default/files/whyasthma-still-kills-full-report.pdf

10. Suissa S, Ernst P, Benayoun S, Baltzan M, Cai B. Low-dose inhaled corticosteroids and the prevention of death from asthma. N Engl J Med. 2000;343: 332-6.

11. Sadatsafavi M, Lynd LD, Marra CA, FitzGerald JM. Dispensation of long-acting $\beta$ agonists with or without inhaled corticosteroids, and risk of asthma-related hospitalisation: a population-based study. Thorax. 2014;69:328-34.

12. Gershon AS, Campitelli MA, Croxford R et al., Combination long-acting $\beta$-agonists and inhaled corticosteroids compared with long-acting $\beta$-agonists alone in older adults with chronic obstructive pulmonary disease. JAMA. 2014;312:1114-21.

13. Schunemann HJ, Jaeschke R, Cook DJ et al., An official ATS statement: grading the quality of evidence and strength of recommendations in ATS guidelines and recommendations. Am J Respir Crit Care Med. 2006;174:605-14.

14. National Asthma Council Australia Australian Asthma Handbook, V1.3. 2017. 
15. Keeley D and Baxter N. Conflicting asthma guidelines cause confusion in primary care. BMJ. 2018;360.

16. Schoenberg NC, Barker AF, Bernardo J et al., A comparative analysis of pulmonary and critical care medicine guideline development methodologies. Am J Respir Crit Care Med.2017;196:621-7.

17. Krishnan JA. Developing Clinical Guidelines: 99\% Faster Is Not Enough. Am J Respir Crit Care Med. 2017;196:543-4.

18. Travers J, Marsh S, Williams M et al. External validity of randomised controlled trials in asthma: to whom do the results of the trials apply? Thorax. 2007;62:219-23.

19. Roche N, Reddel HK, Agusti A et al., Integrating real-life studies in the global therapeutic research framework. Lancet Respir Med. 2013;1: e29-e30.

20. Travers J, Marsh S, Caldwell B et al., External validity of randomized controlled trials in COPD. Respir Med. 2007;101:1313-20.

21. American Thoracic Society. Standards for the diagnosis and care of patients with chronic obstructive pulmonary disease. Am J Respir Crit Care Med. 1995;152: S77-121.

22. Bateman ED, Reddel HK, van Zyl-Smit RN, Agusti A. The asthma-COPD overlap syndrome: towards a revised taxonomy of chronic airways diseases? Lancet Respir Med. 2015;3:719-28.

23. Reddel HK. Treatment of overlapping asthma-chronic obstructive pulmonary disease: Can guidelines contribute in an evidence-free zone? J Allergy Clin Immunol. 2015;136:546-52.

24. Woodruff PG, van den Berge M, Boucher RC et al., American Thoracic Society/National Heart, Lung, and Blood Institute Asthma-Chronic Obstructive Pulmonary Disease Overlap Workshop Report. Am J Respir Crit Care Med. 2017;196:375-81.

25. Alshabanat A, Zafari Z, Albanyan O, Dairi M, FitzGerald JM. Asthma and COPD Overlap Syndrome (ACOS): A Systematic Review and Meta Analysis. PLoS One. 2015;10:e0136065.

26. Louie S, Zeki AA, Schivo M et al., The asthma-chronic obstructive pulmonary disease overlap syndrome: pharmacotherapeutic considerations. Expert Rev Clin Pharmacol. 2013;6:197-219.

27. Suissa S, Ernst P, Kezouh A. Regular use of inhaled corticosteroids and the long term prevention of hospitalisation for asthma. Thorax. 2002;57:880-4.

28. Gibson PG, Simpson JL. The overlap syndrome of asthma and COPD: what are its features and how important is it? Thorax. 2009;64:728-35.

29. Lau EMT, Roche NA, Reddel HK. Therapeutic approaches to asthma-chronic obstructive pulmonary disease overlap. Expert Rev Clin Immunol. 2017; 13:449-55.

30. Boulet L-P, Vervloet D, Magar Y, Foster JM. Adherence: the goal to control asthma. Clin Chest Med. 2012;33:405-17.

31. AIHW : Correll PK, Poulos LM et al. Respiratory medication use in Australia 2003-2013: treatment of asthma and COPD. 2015. Canberra: AIHW. https://www.aihw.gov.au/reports/chronic-respiratory-conditions/respiratory-medication-use-in-australia-2003-2013.

32. Bender BG, Pedan A and Varasteh LT. Adherence and persistence with fluticasone propionate/salmeterol combination therapy. J Allergy Clin Immunol. 2006;118:899-904.

33. Partridge MR, van der Molen T, Myrseth SE, Busse WW. Attitudes and actions of asthma patients on regular maintenance therapy: the INSPIRE study. BMC Pulm. Med. 2006;6:13.

34. FitzGerald JM, Becker A, Sears MR et al., Doubling the dose of budesonide versus maintenance treatment in asthma exacerbations. Thorax. 2004;59:550-6.

35. Harrison TW, Oborne J, Newton S, Tattersfield AE. Doubling the dose of inhaled corticosteroid to prevent asthma exacerbations: randomised controlled trial. Lancet. 2004;363:271-5.

36. Singh AM, Busse WW. Asthma exacerbations 2: Aetiology. Thorax. 2006;61:809-16.

37. Gibson PG, Powell H, Written action plans for asthma: an evidence-based review of the key components. Thorax. 2004;59:94-9.

38. Quon BS, Fitzgerald JM, Lemiere C, Shahidi N, Ducharme FM. Increased versus stable doses of inhaled corticosteroids for exacerbations of chronic asthma in adults and children. Cochrane Database Syst Rev. 2010;10:CD007524.
39. Cates CJ, Karner C. Combination formoterol and budesonide as maintenance and reliever therapy versus current best practice (including inhaled steroid maintenance), for chronic asthma in adults and children. Cochrane Database Syst Rev. 2013;4:CD007313.

40. Slater MG, Pavord ID, Shaw DE. Step 4: stick or twist? A review of asthma therapy. BMJ Open Respir Research. 2016;3:e000143.

41. Jackson DJ, Bacharier LB, Mauger DT et al., Quintupling inhaled glucocorticoids to prevent childhood asthma exacerbations. N Engl J Med. 2018;378:891-901.

42. McKeever T, Mortimer K, Wilson A et al., Quadrupling inhaled glucocorticoid dose to abort asthma exacerbations. N Engl J Med. 2018;378:902-10.

43. FitzGerald JM, Poureslami I. The need for humanomics in the era of genomics and the challenge of chronic disease management. Chest. 2014;146:10-2.

44. Loymans RJB, Honkoop PJ, Termeer EH. Identifying patients at risk for severe exacerbations of asthma: development and external validation of a multivariable prediction model. Thorax. 2016;71:838-46.

45. Loymans RJB, Debray TPA, Honkoop PJ. Exacerbations in adults with asthma: A systematic review and external validation of prediction models. J Allergy Clin Immunol Pract. 2018.

46. Haldar P, Pavord ID, Shaw DE et al. Cluster analysis and clinical asthma phenotypes. Am J Respir Crit Care Med. 2008;178:218-24.

47. Petsky HL, Cates CJ, Kew KM, Chang AB. Tailoring asthma treatment on eosinophilic markers (exhaled nitric oxide or sputum eosinophils): a systematic review and meta-analysis. Thorax. 2018.

48. Korevaar DA, Westerhof GA, Wang J. Diagnostic accuracy of minimally invasive markers for detection of airway eosinophilia in asthma: a systematic review and meta-analysis. Lancet Respir Med. 2015;3:290-300.

49. McNicholl DM, Stevenson M, McGarvey LP, Heaney LG, The utility of fractional exhaled nitric oxide suppression in the identification of nonadherence in difficult asthma. Am J Respir Crit Care Med. 2012;186:1102-8.

50. Pauwels RA, Pedersen S, Busse WW et al., Early intervention with budesonide in mild persistent asthma: a randomised, double-blind trial. Lancet. 2003;361:1071-6.

51. Reddel HK, Busse WW, Pedersen S et al. Should recommendations about starting inhaled corticosteroid treatment for mild asthma be based on symptom frequency: a post-hoc efficacy analysis of the START study. Lancet. 2017;389:157-66.

52. Dusser D, Montani D, Chanez P et al., Mild asthma: an expert review on epidemiology, clinical characteristics and treatment recommendations. Allergy. 2007;62:591-604.

53. Bateman ED, Reddel HK, O'Byrne PM et al., As-needed budesonide-formoterol versus maintenance budesonide in mild asthma. N Engl J Med. 2018;378:1877-87.

54. O'Byrne PM, FitzGerald JM, Bateman ED et al., Inhaled combined budesonide-formoterol as needed in mild asthma. N Engl J Med. 2018; 378:1865-76.

55. Smith AD, Cowan JO, Brassett KP et al. Exhaled nitric oxide: a predictor of steroid response. Am J Respir Crit Care Med. 2005;172:453-9.

56. Price DB, Buhl R, Chan A et al., Fractional exhaled nitric oxide as a predictor of response to inhaled corticosteroids in patients with non-specific respiratory symptoms and insignificant bronchodilator reversibility: a randomised controlled trial. Lancet Respir Med. 2018;6:29-39.

57. Lai K, Liu B, Xu D et al., Will nonasthmatic eosinophilic bronchitis develop into chronic airway obstruction?: a prospective, observational study. Chest. 2015;148:887-94.

58. Dekhuijzen PNR. Guidance on handheld inhalers in asthma and COPD guidelines: An update. Respir Med. 2015;109:1592-3.

59. de Vries R, Dagelet YWF, Spoor P et al. Clinical and inflammatory phenotyping by breathomics in chronic airway diseases irrespective of the diagnostic label. Eur Respir J. 2018;51:1701817.

60. Hekking PP, Wener RR, Amelink $M$ et al., The prevalence of severe refractory asthma. J Allergy Clin Immunol. 2015;135:896-902.

61. Petsky HL, Kew KM, Turner C, Chang AB, Exhaled nitric oxide levels to guide treatment for adults with asthma. Cochrane Database Syst Rev. 2016;9:Cd011440. 